Conclusions The results show that with adequate preventive measures cancer surgeries can be performed with low risk of severe complications and post-surgical COVID positivity.

\section{EPV167/\#371 E-LEARNING IN GYNECOLOGIC ONCOLOGY: A NEW APPROACH FOR MEDICAL STUDENTS DURING COVID 19 PANDEMIC}

${ }^{1} \mathrm{MA}$ Feriaoui, ${ }^{2} \mathrm{R}$ Arfaoui, ${ }^{3,4} \mathrm{Y}$ Berrazega, ${ }^{5} \mathrm{~S}$ Khedhri, ${ }^{2} \mathrm{M}$ Laamiri, ${ }^{2} \mathrm{G}$ Khouildi, ${ }^{2} \mathrm{~K}$ Hajiami, ${ }^{1} \mathrm{O}$ Benzineb, ${ }^{5} \mathrm{M}$ Malek, ${ }^{5} \mathrm{~K}$ Neji. ${ }^{1}$ Tunis maternity center, Department B of Gynecologic Surgery, Tunis, Tunisia; ${ }^{2}$ Tunis military hospital, Department of Gynecology and Obstetrics, Tunis, Tunisia; ${ }^{3}$ University hospital Abderrahmen Mami Ariana, Medical Oncology, Tunis, Tunisia; ${ }^{4}$ Abderrahmen mami university hospital, Department Medical Oncology, Tunis, Tunisia; ${ }^{5}$ Tunis Maternity Center, Department B of Gynecologic Surgery, Tunis, Tunisia

\subsection{6/ijgc-2021-IGCS.237}

Objectives Since the worldwide spread of Covid 19 pandemic, medical education is affected. To ensure the integrity and the continuity of medical education e-learning has been adopted.

Methods To evaluate the efficiency of e-learning in gynecologic oncology education in time of Covid 19 pandemic, we do a comparative study including 30 undergraduate medical students. Half of them received traditional learning about four gynecologic cancer (endometrial, ovarian, cervical and breast cancers), the others received an e-learning education for the same chapters. Clinical knowledge was evaluated before and after getting the courses in the two groups.

Results There wasn't a significant differences comparing the two groups evaluations. Before getting the endometrial cancer course, $74 \%$ of e-learning group students (first group) have less than the average score $(5 / 10)$, versus $68 \%$ in the groupe of traditional learning (the second group). By receiving the course, the rate of good response (more than 5/10) increases respectively to 92 and $94 \%$. Similar rates were reported for the other delivered chapters.

Conclusions Based on the non significant differences between results of the two learning methods, e-learning is a efficient tool to provide gynecologic oncology education in time of Covid 19 pandemic. More studies are needed to evaluate the implementation and the student's adherence to this educational process.

\section{EPV168/\#474 MEDICAL CARE OF PATIENTS WITH GYNECOLOGIC CANCER DURING THE COVID-19 PANDEMIC: EXPERIENCE OF A CANCER CENTER IN BRAZIL}

${ }^{1}$ A Moura*, 'MT Branco, ${ }^{1} \mathrm{PH}$ Oliveira, ${ }^{1} \mathrm{M}$ Pavoni, ${ }^{2} \mathrm{~A}$ Vanzo, ${ }^{3} \mathrm{~V}$ De Oliveira, ${ }^{1} \mathrm{~A}$ Barros Junior, ${ }^{4}$ S Santos. 'Brasilia's Catholic University, Medicine, Brasilia, Brazil; ${ }^{2}$ Brasilia's University Hospital, Medical Oncology, Brasilia, Brazil; ${ }^{3}$ Brasilia's University Hospital, Gynecologic Oncology, Brasilia, Brazil; ${ }^{4}$ Hospital Santa Lucia, Oncology, Brasilia, Brazil

\subsection{6/ijgc-2021-IGCS.238}

Objectives The objective is to assess the impact of COVID-19 pandemic on the care of gynecologic oncology patients.

Methods This is a retrospective cohort study of all gynecology oncology new patients treated at Brasilia's University Hospital - Brazil. We compared to periods: pre-COVID-19 (March 2019 to February 2020) and during COVID-19 pandemic (March 2020 to February 2021).
Results There was a 53\% reduction in patients with gynecological cancer undergoing treatment at our hospital. The total of surgeries performed was 40 pre and 18 during the pandemic period, a reduction in the surgical volume by $55 \%$. The most operated tumor in the pre-pandemic period was cervix and during was an equal number of surgeries for cervix, endometrial and ovarian cancer. Admissions for chemotherapy or radiotherapy also decreased by 52\%. A total of 78 patients underwent cancer treatment before and 37 during the pandemic. The most frequently treated tumor is cervical cancer, with 53 cases in the pre and 26 during the pandemic, followed by ovarian cancer with 14 cases before and only two during the same period. The median waiting time between diagnosis and surgery was 4.44 days longer during the pandemic, as well as the median time to start chemo and radiotherapy was also longer during the pandemic period.

Conclusions We observed a significant decrease in the number of gynecological cancer patients undergoing treatment during the COVID-19 pandemic. Our results will help health professionals to understand the indirect consequences of the pandemic and the role of women's health care services in minimizing these consequences.

\section{EPV169/\#639 ANXIETY AND DEPRESSION IN BREAST CANCER PATIENTS DURING COVID-19 PANDEMIC IN TUNISIA}

${ }^{1} \mathrm{Y}$ Berrazaga*, 'H Rachdi, ${ }^{1} \mathrm{~N}$ Mejri, ${ }^{2} \mathrm{M}$ Ferjaoui, ${ }^{1} \mathrm{~N}$ Daoud, ${ }^{1} \mathrm{H}$ Boussen. ${ }^{1} \mathrm{Abdrahman}$ Mami Hospital Medical Oncology Department Tunisia, Medical Oncology, Ariana, Tunisia; ${ }^{2}$ Maternity and Neonatal Center of Tunis, B, Tunis, Tunisia

\subsection{6/ijgc-2021-IGCS.239}

Objectives The COVID-19 pandemic has generated global mental health crisis. It has resulted in new sources of anxiety and depression among breast cancer patients. This study aimed to evaluate the anxiety and depression in Tunisian breast cancer patients.

Methods We conducted an observational study between January and May 2021 during the COVID-19 pandemic. Symptoms of anxiety and depression in cancer patients were screened using the Hospital Anxiety and Depression Scale (HADS). We present preliminary results of a large study. Twenty patients replied to this survey until now.

Results Mean age of interviewed patients was 47 years [30-67 years]. Eleven patients (55\%) had metastatic disease. We reported 14 complete/partial responses (70\%), 3 stable diseases $(15 \%)$ and 3 progressive diseases (15\%). About marital status, $14(70 \%)$ were married, 2 (10\%) divorced, 41(5\%) widowed and $3(15 \%)$ were single. Five women $(25 \%)$ wanted to see a psychiatrist. The incidence of depression was 35\% (7/20). Six patients $(86 \%)$ had mild depression and one patient $(14 \%)$ moderate depression. The incidence of anxiety was 25\% (5/ $20)$. of those patients, $3(60 \%)$ were experiencing mild anxiety, one patient (20\%) moderate anxiety and one (20\%) severe anxiety. There were no correlations between anxiety or depression and age, educational or socioeconomic level, marital status, breast cancer stage and treatment delays during COVID.

Conclusions This study showed high rates of depression and anxiety during the COVID-19 pandemic. A psychological care should be offered to breast cancer patients. 